\title{
Stream Division by a Channel Bottom Orifice
}

\author{
Jerzy M. Sawicki, Michał Siebert \\ Gdańsk University of Technology, Faculty of Civil and Environmental Engineering, ul. Narutowicza 11/12, \\ 80-233 Gdańsk, Poland, e-mails: jsaw@pg.gda.pl, michalsiebert@gmail.com
}

(Received April 26, 2012; revised July 27, 2012)

\begin{abstract}
A particularly important application of devices dividing a stream of liquid is in storm water drainage systems. Dividers of rainwater sewage streams have in recent years aroused considerable interest among producers of separators. It is therefore important to develop mathematically simple and, if possible, physically precise methods of sizing such structures. It is all the more important given that there are few such methods in the literature on hydraulics. This work is meant to partially remedy this deficiency by elaborating on the calculation methodology and by verifying it empirically. A circular orifice at the bottom of the supplying channel is technically the simplest type of stream divider (which makes it higly practical and particularly appealing to producers). A procedure for dimensioning the structure under consideration is proposed here. It is formally simple, being meant as a practical designing tool. This proposal has been verified experimentally. The degree of the division of the stream was measured in a laboratory model. The main issues were the stability of the characteristics of the lateral outflow and the possibility of applying the classical equations of hydraulics to size the type of divider considered here. In both cases the results obtained may be regarded as positive. Admittedly, the output of the lateral outflow exceeds the nominal value for greater inflows $Q_{p}$, but it is at most double the nominal value. From a technical point of view, this may be regarded as a good result, considering the simplicity of the system. The answer to the second issue proved even more satisfactory. It turns out that the formally simple hydraulics relationships provide a technically consistency of theory with measurement results. Thus the designing of objective devices does not require the application of difficult, time-consuming and costly methodology.
\end{abstract}

Key words: flow regulator, overfall, storm sewer

\section{Notation}

$b \quad-$ width of the overfall edge,

$d-$ diameter of the orifice,

$D$ - diameter of the main channel,

$D_{R i}$ - degree of the stream division (division ratio),

$F \quad-$ orifice cross-sectional area,

$g$ - gravitational acceleration,

$h_{o} \quad-$ accumulation of water above the opening,

$i_{o} \quad-$ channel bottom slope, 
$n$ - Manning coefficient,

$R_{h}-$ hydraulic radius,

$S$ - cross-section of the stream,

$Q_{k i}$ - outflow output of the $i$-th channel,

$Q_{m}$ - maximal output of the water treatment device,

$Q_{n}-$ nominal output of the water treatment device,

$Q_{p}$ - output of the main channel,

$Q_{s}-$ current output of the orifice,

$\mu_{o}-$ coefficient of the orifice output,

$\mu_{p}-$ coefficient of the overflow output.

\section{General Remarks}

The division of a liquid stream into two (or more) parts is a frequent element of hydraulic systems that is very simple structurally as well as extremely important. Its basic quantitative index is the division ratio:

$$
D_{R i}=\frac{Q_{k i}}{Q_{p}},
$$

where $i=1 \ldots I$, and $I$ is the number of partial streams.

In functional terms, it is worth noting two tasks connected with the practical aspects of distributive systems:

- determining the degree of division for an existing system (utilisation task);

- constructing new systems for a given degree of stream division (designing task).

In technical terms, one is dealing with a great variety of specific structures. Classifying them would be superfluous, but it is expedient to mark several important aspects of such hydraulic structures, such as:

- natural systems (rivers, streams);

- technical systems.

Depending on the method of classification we have:

- automatic systems (their functioning is determined by the geometry of the system and its hydraulic properties);

- constrained systems.

With respect to the scale of the systems, it is possible to distinguish:

- 'point' and 'linear' systems (a typical example being water supply pipelines and nodes, whose degree of division may be calculated by using the standard coefficients of local and linear losses); 
- 'cubature' systems (the dimensions and shape of which should be taken into account in calculations).

In terms of technical construction, there are:

- mobile distributors (e.g. floats, mobile troughs);

- immobile distributors (e.g. lateral overflow).

Another important aspect of a system is the temporal variability of its characteristic, which may be:

- stationary (with a constant degree of division);

- non-stationary (with a variable degree of division).

It must be noted that the aspects listed above are independent of each other. Thus they form a large number of combinations, although it may not be possible to indicate a technical justification or an example for each of them.

\section{Aim of the Paper}

A particularly important application of devices dividing a stream of liquid is in storm water drainage systems. These are distinguished by their specific attributes. In the early stages of precipitation, when its intensity is small, rainwater sewage is heavily polluted, and should therefore be entirely directed to sewage treatment devices. However, in later stages of precipitation, with its greater intensity, the concentration of pollution in sewage decreases. In order to avoid overusing sewage treatment devices, it is expedient to divide the stream of inflowing sewage. The part corresponding to the nominal output of the structures concerned, $Q_{n}$, undergoes clarification, while the excess $\left(Q_{p}-Q_{n}\right)$ is conducted directly to the collector. At this stage the notion of the maximal output, $Q_{m}$, appears. In Polish conditions it is accepted that:

$$
Q_{m}=10 Q_{n} .
$$

The issue in question is particularly important for separators of mechanical impurities, which are widely used in systems draining transport thoroughfares and nodes (streets, highways, roads, motorways, car parks, vehicle manoeuvre areas etc.). These are in fact small sediment traps (for granular suspensions) or floatation devices (for oil-derivative substances). They function as a rule under difficult conditions, far from habitation, and therefore have to be stable and reliable, as well as cheap.

This means that the systems dividing a stream of rainwater sewage $\left(Q_{p}\right)$ into the output directed into the separator $\left(Q_{s} \leq Q_{n}\right)$ and the output omitting the separator $\left(Q_{p}-Q_{n}\right.$, when $\left.Q_{p}>Q_{n}\right)$ must be simple and small (so that they could fit inside a separator and not require an individual structure, which would raise the cost of the system). An additional difficulty is the fact that separators are equipped with different 


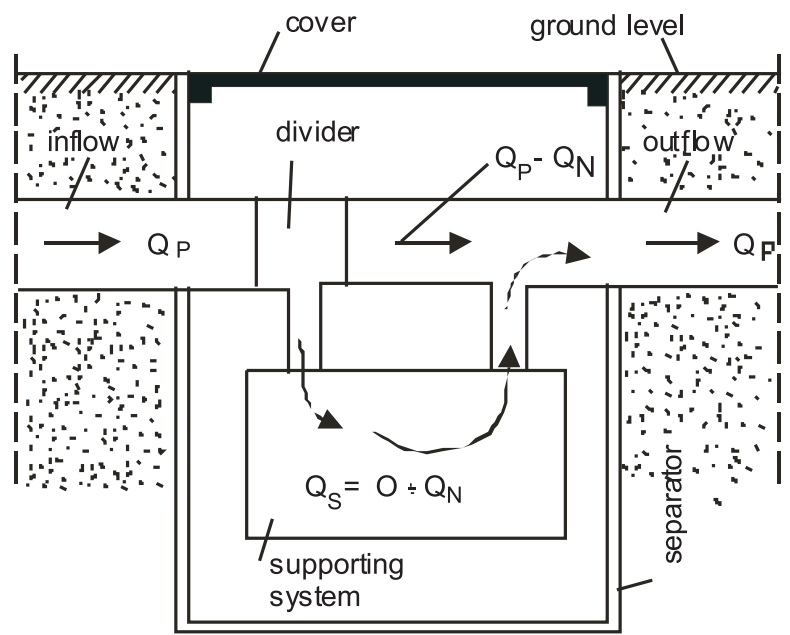

Fig. 1. Schematic diagram of a separator

systems assisting the removal of suspensions (mainly coalescers or high-efficiency settling packets (Ecol 2009)). An outline of such a structure is shown in Fig. 1.

It is worth noting that dividers of rainwater sewage streams (colloquially known as 'by-passes') have in recent years aroused considerable interest among producers of separators. It is therefore important to develop mathematically simple and, if possible, physically precise methods of sizing such structures. It is all the more important given that such methods are lacking in the literature on hydraulics. This work is meant to partially remedy this deficiency by elaborating on the calculation methodology and by verifying it empirically.

\section{Subject of the Investigation}

In this work a circular orifice at the bottom of the supplying channel is considered. It is technically the simplest type of stream divider (which makes it highly practical and particularly appealing to producers). In some variants the channel is equipped with a bottom threshold, which acts as a local overflow and serves the purpose of blocking a lower than nominal flow and directing it into the orifice. Schematic longitudinal sections of the systems under discussion are shown in Fig. 2.

\section{Scope of the Investigation}

In the theoretical section of this work a procedure for dimensioning the structure under consideration is proposed. It is formally simple, being meant as a practical designing tool. This proposal has been verified experimentally. The results are shown in further sections of this paper. For this purpose, the degree of the division of the stream was measured in a laboratory model. 


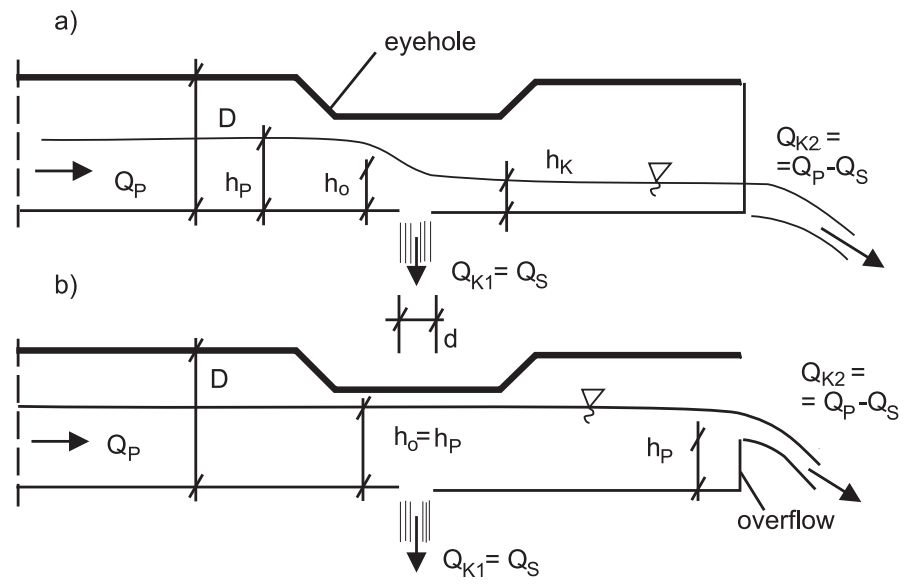

Fig. 2. Longitudinal sections of the divider a) without overflow, b) with overflow

\section{Computational Procedure}

The type of liquid stream divider analysed here consists in a hydraulic intake of the compression of two (Fig. 2a) or three (Fig. 2b) basic flow structures.

In the first case, it is a compressed system of an open channel and an orifice in the horizontal partition (i.e. in the bottom of the channel). This means that the following two formulas are available:

- the Manning formula (Sawicki 2009):

$$
Q_{p}=\frac{1}{n} R_{H}^{2 / 3} i_{o}^{1 / 2} S,
$$

- the formula describing the output of the opening:

$$
Q_{S}=\mu F \sqrt{2 g h_{o}} .
$$

As a reminder, the degree of the division of the stream is being investigated. In this case, it is sufficient to calculate it for the opening, which according to Eq. (1) can be expressed as follows:

$$
D_{R s}=\frac{Q_{S}}{Q_{p}} .
$$

The quantities $R_{h}$ and $S$ in Eq. (3) are, for instance, functions of the maximal (for the current output $Q_{p}$ ) depth of the channel $\left(h_{p}\right.$ in Fig. 2$)$. In a precise attitude one should take into account that, as a result of the outflow of part of the water in the ramification, the depth of the stream will somewhat decrease, attaining the value $h_{k}$ in the outflowing part of the current, while the accumulation above the orifice $h_{o}$ will also have an intermediate value between $h_{p}$ and $h_{k}$. 
Unfortunately, it is impossible to calculate the change in depth in a technically acceptable way. Therefore in the model proposed it has been assumed that:

$$
h_{p}=h_{o}
$$

Thus the calculation procedure consists of three stages:

1. For the accepted discharge of the inflowing stream $Q_{p}$ (with the values $h_{p}, D$ and $i_{o}$ under consideration), the value of $h_{o}=h_{p}$ is calculated from Eq. (3) (owing to the complex form of the relationship, the supplementation of $h_{p}$ is marked out from the characteristics of the channel flow (Sawicki 2009)).

2. The output of the orifice is calculated from Eq. (4).

3. The degree of the division of the stream for the given $Q_{p}$ is calculated from Eq. (5). This makes it possible to obtain the function $D_{R s}\left(Q_{p}\right)$ for a changing inflowing output.

In the case of a channel equipped with a threshold (Fig. 2b) the situation is somewhat different: there is a combination of the channel, orifice and overflow. However, since the conditions of the flow are determined by the accumulation of water above the edge of the overflow, it is enough to use the equation for the output of an overflow with a sharp edge (Sawicki 2009), which in this case takes the form of (Fig. 2b):

$$
Q_{p}-Q_{s}=\frac{2}{3} \mu_{p} b \sqrt{2 g}\left(h_{p}-H_{p}\right)^{3 / 2} .
$$

The insertion of the value $Q_{s}$, described by Eq. (4) for $h_{o}=h_{p}$, into the equation produces the complex relationship $h_{p}\left(Q_{p}\right)$ :

$$
Q_{p}=\mu F \sqrt{2 g h_{p}}+\frac{2}{3} \mu_{p} b \sqrt{2 g}\left(h_{p}-H_{p}\right)^{3 / 2} .
$$

This makes it possible to adopt the following procedure:

1. For the accepted output $Q_{p}$ (with the values $D, d, b$ and $H_{p}$ under consideration), the current accumulation over the edge of the overflow $h_{p}$ is calculated from Eq. (8);

2. $Q_{s}$ is calculated from Eq. (4);

3. $D_{R s}\left(Q_{p}\right)$ is calculated from Eq. (5).

\section{Course of the Experiment}

First a channel of diameter $50 \mathrm{~mm}$ was set up, and an orifice of diameter $6 \mathrm{~mm}$ was drilled in it. The measurement channel was set up with a bottom slope of $0.5 \%$. The output of the orifice in the bottom of the pipe was measured with a stop-watch and a graduated measuring utensil. Next the ordinates of the water level before and after the orifice were measured. These procedures were repeated for slopes of $1 \%$ and 
$1.5 \%$. Then the diameter of the orifice was increased to $10 \mathrm{~mm}$, and the same measurements were repeated. The largest orifice had a diameter of $16 \mathrm{~mm}$. In this case as well measurements were made for three values of slope and different flows. In the next measurement series a pipe closer with a partition serving as an overflow was placed at the end of a $50 \mathrm{~mm}$ long pipe. The height of the overflow, as measured from the bottom of the channel, was $15 \mathrm{~mm}$.

Next a channel of diameter $32 \mathrm{~mm}$ was set up. First an orifice of diameter $6 \mathrm{~mm}$ and then another, of diameter $10 \mathrm{~mm}$, were drilled in the channel. A pipe closer of 10 $\mathrm{mm}$ in height, serving as an overflow, was set up within. The outputs and ordinates before and after the orifice were measured at different values of the decline.

\section{Discussion of the Results and Conclusions}

Some examples of the calculated and the measured output of the orifice are shown in Fig. 3 and Fig. 4 (for pipe diameter $50 \mathrm{~mm}$, orifice $16 \mathrm{~mm}$ and slope 1.5\%), Fig. 5, Fig. 6 (for pipe diameter $32 \mathrm{~mm}$ with overflow, orifice $16 \mathrm{~mm}$ and slope $0.5 \%$ ).

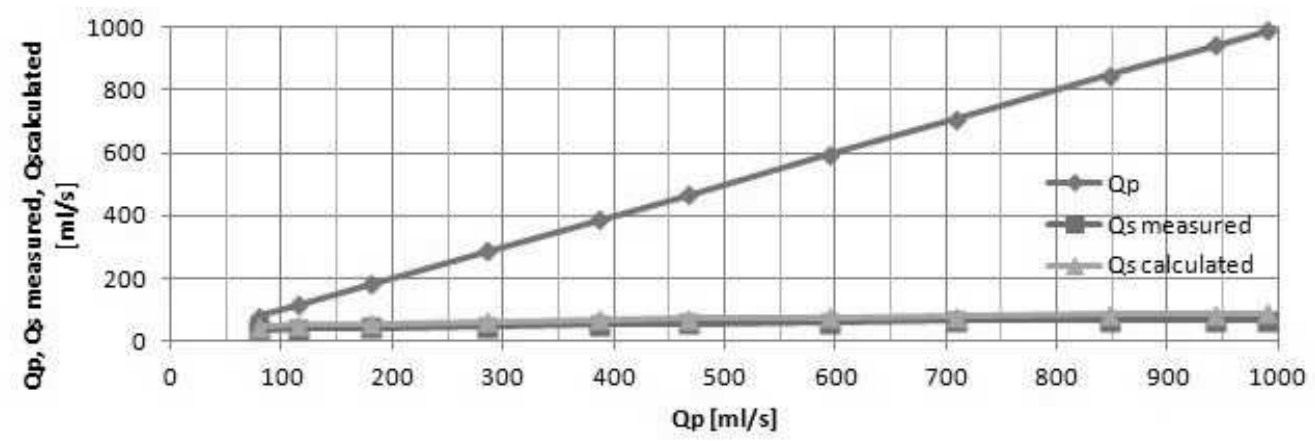

Fig. 3. Main flow and side flow DN $50 \mathrm{~mm}$, orifice $\phi 16 \mathrm{~mm}$, slope $1.5 \%$

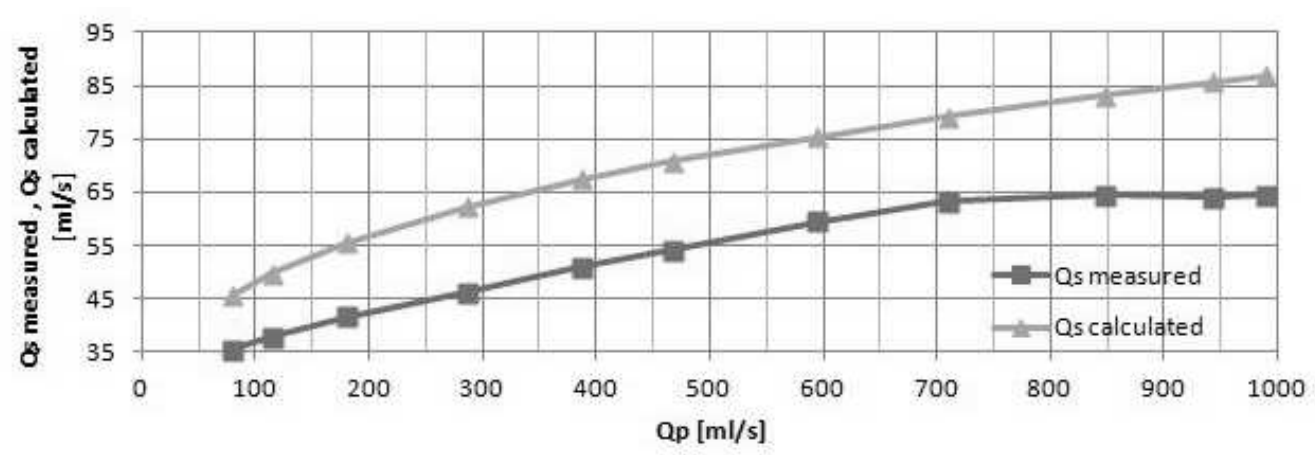

Fig. 4. Measured and calculated side flow DN $50 \mathrm{~mm}$, orifice $\phi 16 \mathrm{~mm}$, slope $1.5 \%$ 


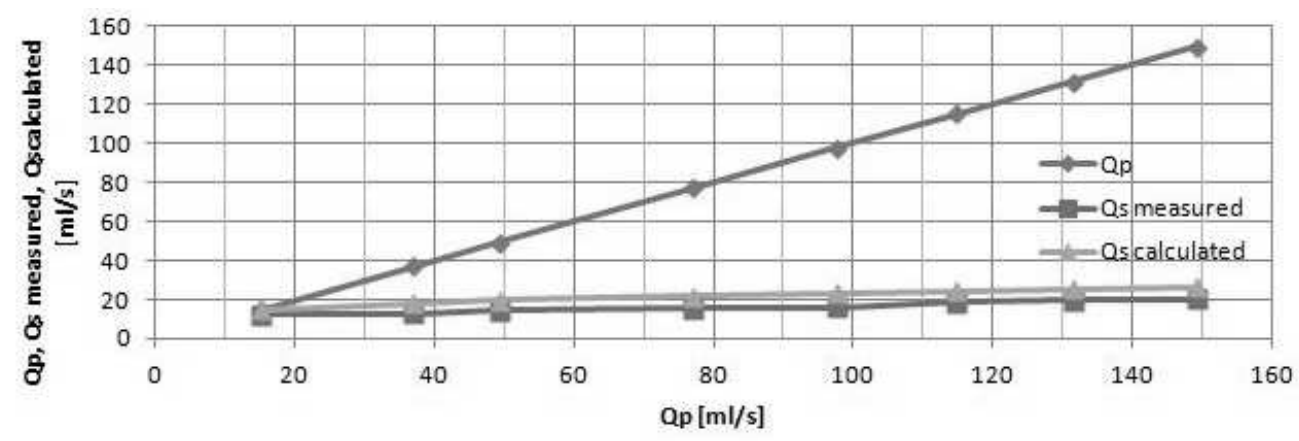

Fig. 5. Main flow and side flow DN $32 \mathrm{~mm}$ with partition, orifice $\phi 10 \mathrm{~mm}$, slope $0.5 \%$

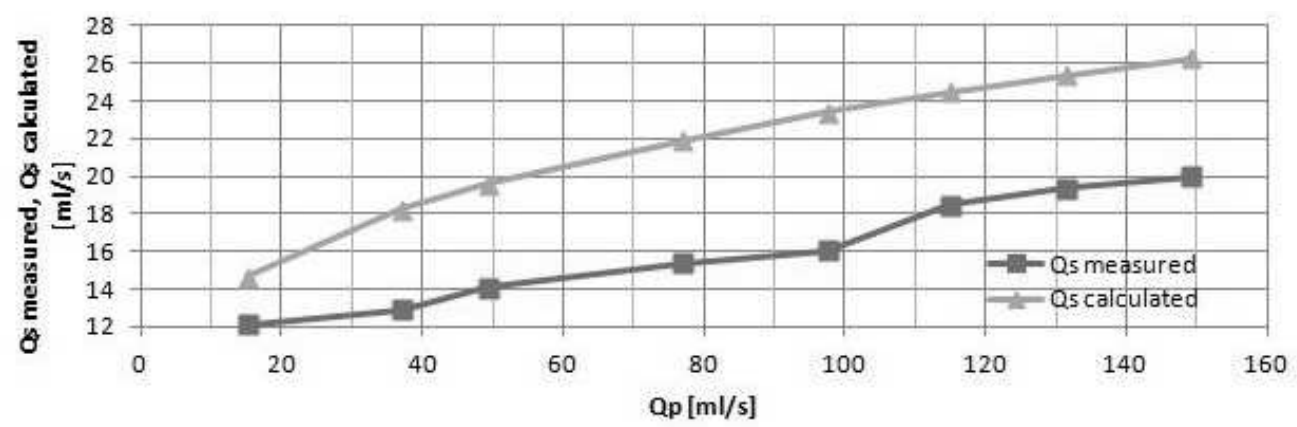

Fig. 6. Measured and calculated side flow DN $32 \mathrm{~mm}$ with partition, orifice $\phi 10 \mathrm{~mm}$, slope $0.5 \%$

The following issues are the main substance of the work:

- The stability of the characteristics of the lateral outflow (in an ideal case, it should take over the whole flow when $Q_{p} \leq Q_{n}$, whereas for $Q_{p}>Q_{n}$ it should direct away a constant value of $Q_{s}=Q_{n}$ );

- The possibility of applying the classical equations of hydraulics for sizing the type of divider considered here.

In both cases the results obtained may be regarded as positive. Admittedly, the output of the lateral outflow exceeds the nominal value for greater inflows $Q_{p}$, but is at most double $\left(Q_{s}\right.$ calculated $=2 Q_{s}$ measured $)$. From a technical point of view, this may be regarded as a good result, considering the simplicity of the system.

The answer to the second issue proved even more satisfactory. It turns out that the formally simple hydraulics relationships provide a technically acceptable consistency of theory with measurement results. Thus the designing of objective devices does not require the application of difficult, time-consuming and costly methodology. The hydraulic characteristics of the system considered here make it impossible to fulfil the second condition. 


\section{Acknowledgements}

The research has been carried out as part of the project "Innovative resources and effective methods of safety improvement and durability of buildings and transport infrastructure in the sustainable development", financed by the European Union from the European Fund for Regional Development under the Operational Programme "Innovative Economy".

\section{References}

Sawicki J. (2009) Mechanics of Flows, Gdańsk Wydawnictwo Politechniki Gdańskiej (in Polish). Ecol Unicon (2009) http://ekol-unicon.com.pl/pl/oferta/produkty_dla_srodowiska/regulatory?PHPSES $\mathrm{SID}=1519918795 \mathrm{~cd} 96 \mathrm{f} 11 \mathrm{~d} 592502 \mathrm{ddb} 01 \mathrm{a} 64$. 